\title{
DUAL OF BASS NUMBERS AND DUALIZING MODULES
}

\author{
MOHAMMAD RAHMANI AND ABDOLJAVAD TAHERIZADEH
}

\begin{abstract}
Let $R$ be a Noetherian ring and let $C$ be a semidualizing $R$-module. In this paper, we impose various conditions on $C$ to be dualizing. For example, as a generalization of $\mathrm{Xu}[22$ Theorem 3.2], we show that $C$ is dualizing if and only if for an $R$-module $M$, the necessary and sufficient condition for $M$ to be $C$-injective is that $\pi_{i}(\mathfrak{p}, M)=0$ for all $\mathfrak{p} \in \operatorname{Spec}(R)$ and all $i \neq$ ht $(\mathfrak{p})$, where $\pi_{i}$ is the invariant dual to the Bass numbers defined by E.Enochs and J.Xu 8 .
\end{abstract}

\section{INTRODUCTION}

Throughout this paper, $R$ is a commutative Noetherian ring with non-zero identity. A finitely generated $R$-module $C$ is semidualizing if the natural homothety map $R \longrightarrow$ $\operatorname{Hom}_{R}(C, C)$ is an isomorphism and $\operatorname{Ext}_{R}^{i}(C, C)=0$ for all $i>0$. Semidualizing modules have been studied by Foxby [9, Vasconcelos [20] and Golod [10] who used the name suitable for these modules. Dualizing complexes, introduced by A.Grothendieck, is a powerful tool for investigating cohomology theories in algebraic geometry. A bounded complex of $R$ modules $D$ with finitely generated homologies is said to be a dualizing complex for $R$, if the natural homothety morphism $R \rightarrow \mathbf{R H o m}_{R}(D, D)$ is quasiisomorphism, and id $\operatorname{Ho}_{R}(D)<\infty$. These notion has been extended to semidualizing complexes by L.W. Christensen [5]. A bounded complex of $R$-modules $C$ with finitely generated homologies is semidualizing for $R$ if the natural homothety morphism $R \rightarrow \mathbf{R H o m}_{R}(C, C)$ is quasiisomorphism. He used these notion to define a new homological dimension for complexes, namely $\mathrm{G}_{C}$-dimension, which is a generalization of Yassemi's G-dimension [23]. The following, is the translation of a part of [5, Proposition 8.4] to the language of modules:

Theorem 1. Let $(R, \mathfrak{m}, k)$ be a Noetherian local ring and let $C$ be a semidualizing $R$ module. The following are equivalent:

(i) $C$ is dualizing.

(ii) $\mathrm{G}_{C}$-dim ${ }_{R}(M)<\infty$ for all finite $R$-modules $M$.

(iii) $\mathrm{G}_{C}-\operatorname{dim}_{R}(k)<\infty$.

In particular, the above theorem recovers [4, 1.4.9]. Note that $k$ is a Cohen-macaulay $R$ module of type 1. R.Takahashi, in [17, Theorem 2.3], replaced the condition G-dim ${ }_{R}(k)<\infty$ in [4, 1.4.9] by weaker conditions and obtained a nice characterization for Gorenstein rings.

2000 Mathematics Subject Classification. 13C05, 13D05, 13D07, $13 \mathrm{H} 10$.

Key words and phrases. Semidualizing modules, dualizing modules, $\mathrm{G}_{C}$-dimension, Bass numbers, dual of Bass numbers, minimal flat resolution, local cohomology. 
Indeed, he showed that $R$ is Gorenstein, provided that either $R$ admits an ideal $I$ of finite Gdimension such that $R / I$ is Gorenstein, or there exists a Cohen-Macaulay $R$-module of type 1 and of finite G-dimension. The following is the main result of section 3, which generalizes Theorem 1 as well as [17, Theorem 2.3]. See Theorem 3.4 below.

Theorem 2. Let $(R, \mathfrak{m})$ be a Noetherian local ring and let $C$ be a semidualizing $R$-module. The following are equivalent:

(i) $C$ is dualizing.

(ii) There exists an ideal $\mathfrak{a}$ with $\mathrm{G}_{C}-\operatorname{dim}_{R}(\mathfrak{a} C)<\infty$ such that $C / \mathfrak{a} C$ is dualizing for $R / \mathfrak{a}$.

(iii) There exists a Cohen-Macaulay $R$-module $M$ with $r_{R}(M)=1$ and $\mathrm{G}_{C}$-dim ${ }_{R}(M)<$ $\infty$.

(iv) $r_{R}(C)=1$ and there exists a Cohen-Macaulay $R$-module $M$ with $\mathrm{G}_{C^{-}} \operatorname{dim}_{R}(M)<$ $\infty$.

E.Enochs et al. [1, solved a long standing conjecture about the existence of flat covers. Indeed, they showed that if $R$ is any ring, then all $R$-modules have flat covers. E.Enochs [6], determined the structure of flat cotorsion modules. Also, E.Enochs and J.Xu [ㅁ, Definition 1.2], defined a new invariant $\pi_{i}$, dual to the Bass numbers, for modules related to flat resolutions. J.Xu [22, studied the minimal injective resolution of flat $R$-modules and minimal flat resolution of injective $R$-modules. He characterized Gorenstein rings in terms of vanishing of Buss numbers of flat modules, and vanishing of dual of Bass numbers of injective modules. More precisely, the following theorem is [22, Theorems 2.1 and 3.2].

Theorem 3. Let $R$ be a Noetherian ring. The following are equivalent:

(i) $R$ is Gorenstein.

(ii) An $R$-module $F$ is flat if and only if $\mu^{i}(\mathfrak{p}, F)=0$ for all $\mathfrak{p} \in \operatorname{Spec}(R)$ whenever $i \neq \operatorname{ht}(\mathfrak{p})$.

(iii) An $R$-module $E$ is injective if and only if $\pi_{i}(\mathfrak{p}, E)=0$ for all $\mathfrak{p} \in \operatorname{Spec}(R)$ whenever $i \neq \operatorname{ht}(\mathfrak{p})$.

In section 4, we give a generalization of Theorem 3. Indeed, in Theorem 4.3, we prove the following result.

Theorem 4. Let $R$ be a Noetherian ring and let $C$ be a semidualizing $R$-module. The following are equivalent:

(i) $C$ is pointwise dualizing.

(ii) An $R$-module $M$ is $C$-injective if and only if $\pi_{i}(\mathfrak{p}, M)=0$ for all $\mathfrak{p} \in \operatorname{Spec}(R)$ whenever $i \neq$ ht $(\mathfrak{p})$.

(iii) An $R$-module $M$ is injective if and only if $\pi_{i}\left(\mathfrak{p}, \operatorname{Hom}_{R}(C, M)\right)=0$ for all $\mathfrak{p} \in$ $\operatorname{Spec}(R)$ whenever $i \neq$ ht $(\mathfrak{p})$.

Theorem 4 has several applications. Let $(R, \mathfrak{m})$ be a $d$-dimensional Cohen-Macaulay local ring possessing a canonical module. In this section, we give the structure of the minimal flat resolution of $\mathrm{H}_{\mathfrak{m}}^{d}(R)$, the top local cohomology of $R$. More precisely, the following theorem is Corollary 4.7 . 
Theorem 5. Let $(R, \mathfrak{m})$ be a $d$-dimensional Cohen-Macaulay local ring possessing a canonical module. The minimal flat resolution of $\mathrm{H}_{\mathfrak{m}}^{d}(R)$ is of the form

$$
0 \longrightarrow \widehat{R_{\mathfrak{m}}} \longrightarrow \cdots \longrightarrow \prod_{\mathrm{ht}(\mathfrak{p})=1} T_{\mathfrak{p}} \longrightarrow \prod_{\mathrm{ht}(\mathfrak{p})=0} T_{\mathfrak{p}} \longrightarrow \mathrm{H}_{\mathfrak{m}}^{d}(R) \longrightarrow 0
$$

in which $T_{\mathfrak{p}}$ is the completion of a free $R_{\mathfrak{p}}$-module with respect to $\mathfrak{p} R_{\mathfrak{p}}$-adic topology.

In this section, by using the above resolution, we obtain the following isomorphism for a $d$-dimensional Cohen-Macaulay local ring (See Corollary 4.8).

$$
\operatorname{Tor}_{i}^{R}\left(\mathrm{H}_{\mathfrak{m}}^{d}(R), \mathrm{H}_{\mathfrak{m}}^{d}(R)\right) \cong\left\{\begin{array}{cl}
\mathrm{H}_{\mathfrak{m}}^{d}(R) & i=d \\
0 & i \neq d
\end{array}\right.
$$

\section{PRELIMINARIES}

In this section, we recall some definitions and facts which are needed throughout this paper. By an injective cogenerator, we always mean an injective $R$-module $E$ for which $\operatorname{Hom}_{R}(M, E) \neq 0$ whenever $M$ is a nonzero $R$-module. For an $R$-module $M$, the injective hull of $M$, is always denoted by $E(M)$.

Definition 2.1. Let $\mathcal{X}$ be a class of $R$-modules and $M$ an $R$-module. An $\mathcal{X}$-resolution of $M$ is a complex of $R$-modules in $\mathcal{X}$ of the form

$$
X=\ldots \longrightarrow X_{n} \stackrel{\partial_{n}^{X}}{\longrightarrow} X_{n-1} \longrightarrow \ldots \longrightarrow X_{1} \stackrel{\partial_{1}^{X}}{\longrightarrow} X_{0} \longrightarrow 0
$$

such that $\mathrm{H}_{0}(X) \cong M$ and $\mathrm{H}_{n}(X)=0$ for all $n \geq 1$. Also the $\mathcal{X}$-projective dimension of $M$ is the quantity

$$
\mathcal{X} \text {-pd }{ }_{R}(M):=\inf \left\{\sup \left\{n \geq 0 \mid X_{n} \neq 0\right\} \mid X \text { is an } \mathcal{X} \text {-resolution of } M\right\}
$$

So that in particular $\mathcal{X}$-pd ${ }_{R}(0)=-\infty$. The modules of $\mathcal{X}$-projective dimension zero are precisely the non-zero modules in $\mathcal{X}$. The terms of $\mathcal{X}$-coresolution and $\mathcal{X}$-id are defined dually.

Definition 2.2. A finitely generated $R$-module $C$ is semidualizing if it satisfies the following conditions:

(i) The natural homothety map $R \longrightarrow \operatorname{Hom}_{R}(C, C)$ is an isomorphism.

(ii) $\operatorname{Ext}_{R}^{i}(C, C)=0$ for all $i>0$.

For example a finitely generated projective $R$-module of rank 1 is semidualizing. If $R$ is Cohen-Macaulay, then an $R$-module $D$ is dualizing if it is semidualizing and that $\operatorname{id}_{R}(D)<\infty$. For example the canonical module of a Cohen-Macaulay local ring, if exists, is dualizing.

Definition 2.3. Following [12, let $C$ be a semidualizing $R$-module. We set

$\mathcal{F}_{C}(R)=$ the subcategory of $R$-modules $C \otimes_{R} F$ where $F$ is a flat $R$-module. $\mathcal{I}_{C}(R)=$ the subcategory of $R$-modules $\operatorname{Hom}_{R}(C, I)$ where $I$ is an injective $R-$ module. 
The $R$-modules in $\mathcal{F}_{C}(R)$ and $\mathcal{I}_{C}(R)$ are called $C$-flat and $C$-injective, respectively. If $C=R$, then it recovers the classes of flat and injective modules, respectively. We use the notations $C$-fd and $C$-id instead of $\mathcal{F}_{C}$-pd and $\mathcal{I}_{C}$-id, respectively.

Proposition 2.4. Let $C$ be a semidualizing $R$-module. Then we have the following:

(i) $\operatorname{Supp}(C)=\operatorname{Spec}(R), \operatorname{dim}(C)=\operatorname{dim}(R)$ and $\operatorname{Ass}(C)=\operatorname{Ass}(R)$.

(ii) If $R \rightarrow S$ is a flat ring homomorphism, then $C \otimes_{R} S$ is a semidualizing $S$-module.

(iii) If $x \in R$ is $R$-regular, then $C / x C$ is a semidualizing $R / x R$-module.

(iv) If, in addition, $R$ is local, then $\operatorname{depth}_{R}(C)=\operatorname{depth}(R)$.

Proof. The parts (i), (ii) and (iii) follow from the definition of semidualizing modules. For (iv), note that an element of $R$ is $R$-regular if and only if it is $C$-regular since $\operatorname{Ass}(C)=$ Ass $(R)$. Now an easy induction yields the equality.

Definition 2.5. Let $C$ be a semidualizing $R$-module. A finitely generated $R$-module $M$ is said to be totally $C$-reflexive if the following conditions are satisfied:

(i) The natural evaluation map $M \longrightarrow \operatorname{Hom}_{R}\left(\operatorname{Hom}_{R}(M, C), C\right)$ is an isomorphism.

(ii) $\operatorname{Ext}_{R}^{i}(M, C)=0=\operatorname{Ext}_{R}^{i}\left(\operatorname{Hom}_{R}(M, C), C\right)$ for all $i>0$.

For an $R$-module $M$, if there exists an exact sequence $0 \rightarrow G_{n} \rightarrow \cdots \rightarrow G_{1} \rightarrow G_{0} \rightarrow M \rightarrow 0$, of $R$-modules such that each $G_{i}$ is totally $C$-reflexive, then we say that $M$ has $\mathrm{G}_{C}$-dimension at most $n$, and write $\mathrm{G}_{C^{-}}-\operatorname{dim} R_{R}(M) \leq n$. If there is no shorter such sequence, we set $\mathrm{G}_{C^{-}}$ $\operatorname{dim}_{R}(M)=n$. Also, if such an integer $n$ does not exist, then we say that $M$ has infinite $\mathrm{G}_{C^{-}}$dimension, and write $\mathrm{G}_{C^{-}}-\operatorname{dim} R_{R}(M)=\infty$.

The next proposition collects basic properties of $\mathrm{G}_{C}$-dimension. For the proof, see [10].

Proposition 2.6. Let $(R, \mathfrak{m})$ be local, $M$ a finitely generated $R$-module and let $C$ be a semidualizing $R$-module. The following statements hold:

(i) If $\mathrm{G}_{C}$ - $\operatorname{dim}_{R}(M)<\infty$, and $x \in \mathfrak{m}$ is $M$-regular, then

$$
\mathrm{G}_{C}-\operatorname{dim}{ }_{R}(M)=\mathrm{G}_{C}-\operatorname{dim} R(M / x M)-1 .
$$

If, also, $x$ is $R$-regular, then

$$
\mathrm{G}_{C^{-}} \operatorname{dim}_{R}(M)=\mathrm{G}_{C / x C^{-}} \operatorname{dim}_{R / x R}(M / x M) .
$$

(ii) If $\mathrm{G}_{C}-\operatorname{dim}_{R}(M)<\infty$ and $x$ is an R-regular element in $\operatorname{Ann}_{R}(M)$, then

$$
\mathrm{G}_{C^{-}} \operatorname{dim}_{R}(M)=\mathrm{G}_{C / x C^{-}}-\operatorname{dim}_{R / x R}(M)+1 .
$$

(iii) Let $0 \rightarrow K \rightarrow L \rightarrow N \rightarrow 0$ be a short exact sequence of $R$-modules. If two of $L, K, N$ are of finite $\mathrm{G}_{C}$-dimension, then so is the third.

(iv) If $\mathrm{G}_{C^{-}} \operatorname{dim}_{R}(M)<\infty$, then

$$
\begin{aligned}
\mathrm{G}_{C-\operatorname{dim}}(M) & =\sup \left\{i \geq 0 \mid \operatorname{Ext}_{R}^{i}(M, C) \neq 0\right\} \\
& =\operatorname{depth}(R)-\operatorname{depth}_{R}(M) .
\end{aligned}
$$

Definition 2.7. Let $C$ be a semidualizing $R$-module. The Auslander class with respect to $C$ is the class $\mathcal{A}_{C}(R)$ of $R$-modules $M$ such that:

(i) $\operatorname{Tor}_{i}^{R}(C, M)=0=\operatorname{Ext}_{R}^{i}\left(C, C \otimes_{R} M\right)$ for all $i \geq 1$, and 
(ii) The natural map $M \rightarrow \operatorname{Hom}_{R}\left(C, C \otimes_{R} M\right)$ is an isomorphism.

The Bass class with respect to $C$ is the class $\mathcal{B}_{C}(R)$ of $R$-modules $M$ such that:

(i) $\operatorname{Ext}_{R}^{i}(C, M)=0=\operatorname{Tor}_{i}^{R}\left(C, \operatorname{Hom}_{R}(C, M)\right)$ for all $i \geq 1$, and

(ii) The natural map $\left.C \otimes_{R} \operatorname{Hom}_{R}(C, M)\right) \rightarrow M$ is an isomorphism.

The class $\mathcal{A}_{C}(R)$ contains all $R$-modules of finite projective dimension and those of finite $C$ injective dimension. Also the class $\mathcal{B}_{C}(R)$ contains all $R$-modules of finite injective dimension and those of finite $C$-projective dimension (see [18, Corollary 2.9]). Also, if any two $R$ modules in a short exact sequence are in $\mathcal{A}_{C}(R)$ (resp. $\mathcal{B}_{C}(R)$ ), then so is the third (see [13]).

Proposition 2.8. Let $(R, \mathfrak{m})$ be a local ring and let $C$ be a semidualizing $R$-module.

(i) $C$ is a dualizing $R$-module if and only if $C \otimes_{R} \widehat{R}$ is a dualizing $\widehat{R}$-module.

(ii) Let $x \in \mathfrak{m}$ be $R$-regular. Then $C$ is a dualizing $R$-module if and only if $C / x C$ is a dualizing $R / x R$-module.

Proof. Just use the definition of dualizing modules.

Theorem 2.9. Let $C$ be a semidualizing $R$-module and let $M$ be an $R$-module.

(i) $C$-id ${ }_{R}(M)=\operatorname{id}_{R}\left(C \otimes_{R} M\right)$ and id $R(M)=C$-id $_{R}\left(\operatorname{Hom}_{R}(C, M)\right)$.

(ii) $C$-fd f $_{R}=\mathrm{fd}_{R}\left(\operatorname{Hom}_{R}(C, M)\right)$ and $\mathrm{fd}_{R}(M)=C$-fd ${ }_{R}\left(C \otimes_{R} M\right)$.

Proof. For (i), see [18, Theorem 2.11] and for (ii), see [19, Proposition 5.2].

Lemma 2.10. Let $C$ be a semidualizing $R$-module, $E$ be an injective cogenerator and $M$ be an $R$-module.

(i) One has $C$-id ${ }_{R}(M)=C$-fd ${ }_{R}\left(\operatorname{Hom}_{R}(M, E)\right)$.

(ii) One has $C$-fd $\mathrm{fd}_{R}(M)=C$-id ${ }_{R}\left(\operatorname{Hom}_{R}(M, E)\right)$.

Proof. (i). We have the following equalities

$$
\begin{aligned}
C-\operatorname{id}_{R}(M) & =\operatorname{id}_{R}\left(C \otimes_{R} M\right) \\
& =\operatorname{fd}_{R}\left(\operatorname{Hom}_{R}\left(C \otimes_{R} M, E\right)\right) \\
& =\operatorname{fd}_{R}\left(\operatorname{Hom}_{R}\left(C, \operatorname{Hom}_{R}(M, E)\right)\right. \\
& =C-\operatorname{fd}_{R}\left(\operatorname{Hom}_{R}(M, E)\right),
\end{aligned}
$$

in which the first equality is from Theorem 2.9(i), and the last one is from Theorem 2.9(ii).

(ii). Is similar to (i).

Remark 2.11. Let $(R, \mathfrak{m})$ be a local ring and let $M$ be a finitely generated $R$-module. We use $\nu_{R}(M)$ to denote the minimal number of generators of $M$. More precisely, $\nu_{R}(M)=$ $\operatorname{vdim}_{R / \mathfrak{m}}\left(M \otimes_{R} R / \mathfrak{m}\right)$. It is easy to see that if $x \in \mathfrak{m}$, then $\nu_{R}(M)=\nu_{R / x R}(M / x M)$. In particular, if $x \in \operatorname{Ann}_{R}(M)$, then $\nu_{R}(M)=\nu_{R / x R}(M)$. Assume that $\operatorname{depth}_{R}(M)=n$. The type of $M$, denoted by $r_{R}(M)$, is defined to be $\operatorname{vdim}_{R / \mathfrak{m}}\left(\operatorname{Ext}_{R}^{n}(R / \mathfrak{m}, M)\right)$. If $x \in \mathfrak{m}$, then $r_{R}(M / x M)=r_{R / x R}(M / x M)$ by [2, Exercise 1.2.26]. Also, if $x \in \mathfrak{m}$ is $M$ - and $R$-regular, then $r_{R}(M)=r_{R / x R}(M / x M)$ by [2, Lemma 3.1.16]. Assuma that $C$ is a semidualizing 
$R$-module. Then $r_{R}(C) \mid r_{R}(R)$. Indeed, by reduction modulo a maximal $R$-sequence, we can assume that $\operatorname{depth}_{R}(C)=0=\operatorname{depth}(R)$. Then we have

$$
\begin{aligned}
r_{R}(R) & =\operatorname{vdim}_{R / \mathfrak{m}} \operatorname{Hom}_{R}(R / \mathfrak{m}, R) \\
& =\operatorname{vdim}_{R / \mathfrak{m}} \operatorname{Hom}_{R}\left(R / \mathfrak{m}, \operatorname{Hom}_{R}(C, C)\right) \\
& =\operatorname{vdim}_{R / \mathfrak{m}} \operatorname{Hom}_{R}\left(R / \mathfrak{m} \otimes_{R} C, C\right) \\
& =\operatorname{vdim}_{R / \mathfrak{m}} \operatorname{Hom}_{R}\left(R / \mathfrak{m} \otimes_{R} C \otimes_{R / \mathfrak{m}} R / \mathfrak{m}, C\right) \\
& =\operatorname{vdim}_{R / \mathfrak{m}} \operatorname{Hom}_{R / \mathfrak{m}}\left(R / \mathfrak{m} \otimes_{R} C, \operatorname{Hom}_{R}(R / \mathfrak{m}, C)\right) \\
& =\nu_{R}(C) r_{R}(C) .
\end{aligned}
$$

In particular, if $r_{R}(R)=1$ (e.g. $R$ is Gorenstein local), then $\nu_{R}(C)=1$ and then $C \cong R$.

Definition 2.12. Let $M$ be an $R$-module and let $\mathcal{X}$ be a class of $R$-modules . Following [7, a $\mathcal{X}$-precover of $M$ is a homomorphism $\varphi: X \rightarrow M$, with $X \in \mathcal{X}$, such that every homomorphism $Y \rightarrow M$ with $Y \in \mathcal{X}$, factors through $\phi$; i.e., the homomorphism

$$
\operatorname{Hom}_{R}(Y, \varphi): \operatorname{Hom}_{R}(Y, X) \rightarrow \operatorname{Hom}_{R}(Y, M)
$$

is surjective for each module $Y$ in $\mathcal{X}$. A $\mathcal{X}$-precover $\varphi: X \rightarrow M$ is a $\mathcal{X}$-cover if every $\psi \in \operatorname{Hom}_{R}(X, X)$ with $\varphi \psi=\varphi$ is an automorphism.

Definition 2.13. Following [6], an $R$-module $M$ is called cotorsion if $\operatorname{Ext}_{R}^{1}(F, M)=0$ for any flat $R$-module $\mathrm{F}$.

Remark 2.14. In [1, E. Enochs et al. showed that if $R$ is any ring, then every $R$-module has a flat cover. It is easy to see that flat cover must be surjective. By [6, Lemma 2.2], the kernel of a flat cover is always cotorsion. So that if $F \rightarrow M$ is flat cover and $M$ is cotorsion, then so is $F$. Therefore for an $R$-module $M$, one can iteratively take flat covers to construct a flat resolution of $M$. Since flat cover is unique up to isomorphism, this resolution is unique up to isomorphism of complexes. Such a resolution is called the minimal flat resolution of $M$. Note that the minimal flat resolution of $M$ is a direct summand of any other flat resolution of $M$. Assume that

$$
\cdots \rightarrow F_{i} \rightarrow \cdots \rightarrow F_{1} \rightarrow F_{0} \rightarrow M \rightarrow 0,
$$

is the minimal flat resolution of $M$. Then $F_{i}$ is cotorsion for all $i \geq 1$. If, in addition, $M$ is cotorsion, then all the flat modules in the minimal flat resolution of $M$ are cotorsion. E. Enochs [6], determined the structure of flat cotorsion modules. He showed that if $F$ is flat and cotorsion, then $F \cong \prod_{\mathfrak{p}} T_{\mathfrak{p}}$ where $T_{\mathfrak{p}}$ is the completion of a free $R_{\mathfrak{p}}$-module with respect to $\mathfrak{p} R_{\mathfrak{p}}$-adic topology. So that we can determine the structure of the minimal flat resolution of cotorsion modules.

Definition 2.15. Let $M$ be a cotorsion $R$-module and let

$$
\cdots \rightarrow F_{i} \rightarrow \cdots \rightarrow F_{1} \rightarrow F_{0} \rightarrow M \rightarrow 0,
$$

be the minimal flat resolution of $M$. Following [8], for a prime ideal $\mathfrak{p}$ of $R$ and an integer $i \geq 0$, the invariant $\pi_{i}(\mathfrak{p}, M)$ is defined to be the cardinality of the basis of a free $R_{\mathfrak{p}}$-module whose completion is $T_{\mathfrak{p}}$ in the product $F_{i} \cong \prod_{\mathfrak{p}} T_{\mathfrak{p}}$. By [8, theorem 2.2], for each $i \geq 0$,

$$
\pi_{i}(\mathfrak{p}, M)=\operatorname{vdim}_{R_{\mathfrak{p}} / \mathfrak{p} R_{\mathfrak{p}}} \operatorname{Tor}_{i}^{R_{\mathfrak{p}}}\left(R_{\mathfrak{p}} / \mathfrak{p} R_{\mathfrak{p}}, \operatorname{Hom}_{R}\left(R_{\mathfrak{p}}, M\right)\right) .
$$


Remark 2.16. Let $M$ be a finitely generated $R$-module. There are isomorphisms

$$
\begin{aligned}
\operatorname{Hom}_{R}(M, E(R / \mathfrak{p})) & \cong \operatorname{Hom}_{R}\left(M, E(R / \mathfrak{p}) \otimes_{R} R_{\mathfrak{p}}\right) \\
& \cong \operatorname{Hom}_{R}(M, E(R / \mathfrak{p})) \otimes_{R} R_{\mathfrak{p}} \\
& \cong \operatorname{Hom}_{R_{\mathfrak{p}}}\left(M_{\mathfrak{p}}, E_{R_{\mathfrak{p}}}\left(R_{\mathfrak{p}} / \mathfrak{p} R_{\mathfrak{p}}\right)\right),
\end{aligned}
$$

where the the first isomorphism holds because $E(R / \mathfrak{p}) \cong E_{R_{\mathfrak{p}}}\left(R_{\mathfrak{p}} / \mathfrak{p} R_{\mathfrak{p}}\right)$, and the second isomorphism is tensor-evaluation [7, Theorem 3.2.14].

\section{Finiteness of $\mathrm{G}_{C}$-Dimension}

Throughout this section, $C$ is a semidualizing $R$-module. We begin with three lemmas that are needed for the main result of this section. It is well-known that a local ring over which there exists a non-zero finitely generated injective module, must be Artinian. Our first lemma generalizes this fact by replacing the injectivity condition with weaker assumption.

Lemma 3.1. Let $(R, \mathfrak{m})$ be local and let $M$ be a finitely generated $R$-module with $\operatorname{depth}(M)=$ 0. If $\operatorname{Ext}_{R}^{1}(R / \mathfrak{m}, M)=0$, then $R$ is Artinian. In particular, $M$ is injective.

Proof. We show that $\operatorname{dim}(R)=0$. Assume, on the contrary, that $\operatorname{dim}(R)>0$. Note that if $N$ is an $R$-module of finite length, then by using a composition series for $N$ in conjunction with the assumption, we have $\operatorname{Ext}_{R}^{1}(N, M)=0$. Now an easy induction on $\ell_{R}(N)$ yields the equality $\ell_{R}\left(\operatorname{Hom}_{R}(N, M)\right)=\ell_{R}(N) \ell_{R}\left(\operatorname{Hom}_{R}(R / \mathfrak{m}, M)\right)$. Next, note that $\ell_{R}\left(R / \mathfrak{m}^{i}\right)<\infty$ for any $i \geq 1$, and that the sequence $\left\{\ell_{R}\left(R / \mathfrak{m}^{i}\right)\right\}_{i=1}^{\infty}$ is not bounded since $\mathfrak{m}^{i} \neq \mathfrak{m}^{i+1}$ for any $i \geq 1$. Hence $\left\{\ell_{R}\left(\operatorname{Hom}_{R}\left(R / \mathfrak{m}^{i}, M\right)\right)\right\}_{i=1}^{\infty}$ is not bounded. But $0:_{M} \mathfrak{m} \subseteq 0:_{M} \mathfrak{m}^{2} \subseteq \cdots$ is a chain of submodules of $M$, and hence is eventually stationary. This is a contradiction. Therefore $R$ is Artinian. Finally, the assumption $\operatorname{Ext}_{R}^{1}(R / \mathfrak{m}, M)=0$ implies that $M$ is injective.

Lemma 3.2. Let $(R, \mathfrak{m})$ be a local ring and let $M$ be a Cohen-Macaulay $R$-module with $\mathrm{G}_{C^{-}}$ $\operatorname{dim}_{R}(M)<\infty$. Then $r_{R}(C) \mid r_{R}(M)$.

Proof. We use induction on $n=\operatorname{depth}(R)$. If $n=0$, then by Proposition 2.6(iv), we have $\mathrm{G}_{C}-\operatorname{dim}_{R}(M)=0$, and hence there is an isomorphism $M \cong \operatorname{Hom}_{R}\left(\operatorname{Hom}_{R}(M, C), C\right)$, and the equalities $\operatorname{depth}_{R}(C)=0=\operatorname{depth}_{R}(M)$. Hence we have

$$
\begin{aligned}
r_{R}(M) & =\operatorname{vdim}_{R / \mathfrak{m}} \operatorname{Hom}_{R}(R / \mathfrak{m}, M) \\
& =\operatorname{vdim}_{R / \mathfrak{m}} \operatorname{Hom}_{R}\left(R / \mathfrak{m}, \operatorname{Hom}_{R}\left(\operatorname{Hom}_{R}(M, C), C\right)\right) \\
& =\operatorname{vdim}_{R / \mathfrak{m}} \operatorname{Hom}_{R}\left(R / \mathfrak{m} \otimes_{R} \operatorname{Hom}_{R}(M, C), C\right) \\
& =\operatorname{vdim}_{R / \mathfrak{m}} \operatorname{Hom}_{R}\left(R / \mathfrak{m} \otimes_{R} \operatorname{Hom}_{R}(M, C) \otimes_{R / \mathfrak{m}} R / \mathfrak{m}, C\right) \\
& =\operatorname{vdim}_{R / \mathfrak{m}} \operatorname{Hom}_{R / \mathfrak{m}}\left(R / \mathfrak{m} \otimes_{R} \operatorname{Hom}_{R}(M, C), \operatorname{Hom}_{R}(R / \mathfrak{m}, C)\right) \\
& =\nu_{R}\left(\operatorname{Hom}_{R}(M, C)\right) r_{R}(C) .
\end{aligned}
$$

Therefore $r_{R}(C) \mid r_{R}(M)$. Now, assume inductively that $n>0$. We consider two cases:

Case 1. If $\operatorname{depth}_{R}(M)=0$, then $M$ is of finite length since it is Cohen-Macaulay. Hence we can take an $R$-regular element $x$ such that $x M=0$. Set $\overline{(-)}=(-) \otimes_{R} R / x R$. Then by 
Proposition 2.6(ii), we have $\mathrm{G}_{\bar{C}}-\operatorname{dim} \bar{R}(M)<\infty$. Also, note that $M$ is a Cohen-Macaulay $\bar{R}$-module. Hence by induction hypothesis we have $r_{\bar{R}}(\bar{C}) \mid r_{\bar{R}}(M)$. Thus $r_{R}(C) \mid r_{R}(M)$.

Case 2. If $\operatorname{depth}_{R}(M)>0$, then we can take an element $y \in \mathfrak{m}$ to be $M$ - and $R$-regular. Set $\overline{(-)}=(-) \otimes_{R} R / y R$. Now $\bar{M}$ is a Cohen-Macaulay $\bar{R}$-module, and that

$$
\mathrm{G}_{\bar{C}^{-}} \operatorname{dim} \bar{R}(\bar{M})=\mathrm{G}_{C^{-}} \operatorname{dim}_{R}(M)<\infty,
$$

by Proposition 2.6(i). Therefore, by induction hypothesis, we have $r_{\bar{R}}(\bar{C}) \mid r_{\bar{R}}(\bar{M})$, whence $r_{R}(C) \mid r_{R}(M)$. This complete the inductive step.

Lemma 3.3. Let $(R, \mathfrak{m})$ be local and that $r_{R}(C)=1$. If there exists a totally $C$-reflexive $R$-module of finite length, then $C$ is dualizing.

Proof. Assume that $M$ is a finite length $C$-reflexive $R$-module. Then $\operatorname{depth}_{R}(M)=0$, and hence $\operatorname{depth}(R)=\mathrm{G}_{C}$ - $\operatorname{dim}(M)=0$ by Proposition 2.6(iv). Therefore, we have $\operatorname{depth}_{R}(C)=0$ by Proposition 2.4(iv). Now assume, on the contrary, that $C$ is not dualizing. Hence, by Lemma 3.1, we have $\operatorname{Ext}_{R}^{1}(R / \mathfrak{m}, C) \neq 0$. Let

$$
0=M_{0} \subset M_{1} \subset \cdots \subset M_{r}=M,
$$

be a composition series for $M$. Thus the factors are all isomorphic to $R / \mathfrak{m}$, and we have exact sequences

$$
0 \rightarrow M_{i-1} \rightarrow M_{i} \rightarrow R / \mathfrak{m} \rightarrow 0
$$

for all $1 \leq i \leq r$. Applying the functor $\operatorname{Hom}_{R}(-, C)$, we get the exact sequence

$$
0 \rightarrow \operatorname{Hom}_{R}(R / \mathfrak{m}, C) \rightarrow \operatorname{Hom}_{R}\left(M_{i}, C\right) \rightarrow \operatorname{Hom}_{R}\left(M_{i-1}, C\right),
$$

for each $1 \leq i \leq r-1$. Now since $\operatorname{depth}_{R}(C)=0$ and $r_{R}(C)=1$, we have $\operatorname{Hom}_{R}(R / \mathfrak{m}, C) \cong$ $R / \mathfrak{m}$. Hence we have the inequality $\ell_{R}\left(\operatorname{Hom}_{R}\left(M_{i}, C\right)\right) \leq \ell_{R}\left(\operatorname{Hom}_{R}\left(M_{i-1}, C\right)\right)+1$ for each $1 \leq i \leq r-1$. On the other hand, application of the functor $\operatorname{Hom}_{R}(-, C)$ on the exact sequence $0 \rightarrow M_{r-1} \rightarrow M \rightarrow R / \mathfrak{m} \rightarrow 0$, yields an exact sequence

$$
\begin{gathered}
0 \rightarrow \operatorname{Hom}_{R}(R / \mathfrak{m}, C) \rightarrow \operatorname{Hom}_{R}(M, C) \rightarrow \operatorname{Hom}_{R}\left(M_{r-1}, C\right) \\
\rightarrow \operatorname{Ext}_{R}^{1}(R / \mathfrak{m}, C) \rightarrow \operatorname{Ext}_{R}^{1}(M, C)=0 .
\end{gathered}
$$

Therefore $\ell_{R}\left(\operatorname{Hom}_{R}(M, C)\right)=\ell_{R}\left(\operatorname{Hom}_{R}\left(M_{r-1}, C\right)\right)+1-\ell_{R}\left(\operatorname{Ext}_{R}^{1}(R / \mathfrak{m}, C)\right)$. But since $\ell_{R}\left(\operatorname{Ext}_{R}^{1}(R / \mathfrak{m}, C)\right)>0$, we have

$$
\begin{aligned}
\ell_{R}\left(\operatorname{Hom}_{R}(M, C)\right) & <\ell_{R}\left(\operatorname{Hom}_{R}\left(M_{r-1}, C\right)\right)+1 \\
& \leq \ell_{R}\left(\operatorname{Hom}_{R}\left(M_{r-2}, C\right)\right)+2 \\
& \leq \cdots \\
& \leq \ell_{R}\left(\operatorname{Hom}_{R}\left(M_{0}, C\right)\right)+r \\
& =r \\
& =\ell_{R}(M) .
\end{aligned}
$$

Now since $\operatorname{Hom}_{R}(M, C)$ is again a totally $C$-reflexive $R$-module of finite length, the same argument shows that $\ell_{R}\left(\operatorname{Hom}_{R}\left(\operatorname{Hom}_{R}(M, C), C\right)\right) \leq \ell_{R}\left(\operatorname{Hom}_{R}(M, C)\right)$. But since $M$ is totally $C$-reflexive, we have $M \cong \operatorname{Hom}_{R}\left(\operatorname{Hom}_{R}(M, C), C\right)$, which implies that $\ell_{R}(M)<$ $\ell_{R}(M)$, a contradiction. Hence $C$ is dualizing.

The following theorem is a generalization of [17, Theorem 2.3].

Theorem 3.4. Let $(R, \mathfrak{m})$ be local. The following are equivalent: 
(i) $C$ is dualizing.

(ii) There exists an ideal $\mathfrak{a}$ with $\mathrm{G}_{C}-\operatorname{dim}_{R}(\mathfrak{a} C)<\infty$ such that $C / \mathfrak{a} C$ is dualizing for $R / \mathfrak{a}$.

(iii) There exists a Cohen-Macaulay $R$-module $M$ with $r_{R}(M)=1$ and $\mathrm{G}_{C}$ - $\operatorname{dim}_{R}(M)<$ $\infty$.

(iv) $r_{R}(C)=1$ and there exists a Cohen-Macaulay $R$-module $M$ of finite $\mathrm{G}_{C}$-dimension.

Proof. (i) $\Longrightarrow$ (ii). Choose $\mathfrak{a}=0$.

(ii) $\Longrightarrow$ (iii). We show that $C / \mathfrak{a} C$ has the desired properties. First, the exact sequence

$$
0 \rightarrow \mathfrak{a} C \rightarrow C \rightarrow C / \mathfrak{a} C \rightarrow 0,
$$

in conjunction with Proposition 2.6(iii), show that $\mathrm{G}_{C}-\operatorname{dim}_{R}(C / \mathfrak{a} C)<\infty$. On the other hand, $C / \mathfrak{a} C$ is a Cohen-Macaulay $R / \mathfrak{a} R$-module and hence is a Cohen-Macaulay $R$-module. Finally, by [2, Exercise 1.2.26], we have $r_{R}(C / \mathfrak{a} C)=r_{R / \mathfrak{a}}(C / \mathfrak{a} C)=1$.

(iii) $\Longrightarrow$ (iv). By Lemma 3.2, we have $r_{R}(C)=1$.

(iv) $\Longrightarrow$ (i). Assume that $M$ is a Cohen-Macaulay $R$-module with $\mathrm{G}_{C}-\operatorname{dim}{ }_{R}(M)<\infty$. We use induction on $m=\operatorname{depth}_{R}(M)$. If $m=0$, then $M$ is of finite length since it is CohenMacaulay. Since $\sqrt{\operatorname{Ann}_{R}(M)}=\mathfrak{m}$, we can choose a maximal $R$-sequence from elements of $\operatorname{Ann}_{R}(M)$, say x. In view of Proposition 2.8(ii) and Proposition 2.6(ii), we can replace $C$ by $C / \mathrm{x} C$ and $R$ by $R / \mathrm{x} R$, and assume that $M$ is totally $C$-reflexive. In this case, $C$ is dualizing by Lemma 3.3. Now assume inductively that $m>0$. Hence $\operatorname{depth}(R)>0$ by Proposition 2.6(iv), and we can take an element $x \in \mathfrak{m}$ to be $M$ - and $R$-regular. Set $\overline{(-)}=(-) \otimes_{R} R / x R$. Now $\bar{M}$ is a Cohen-Macaulay $\bar{R}$-module and $r_{\bar{R}}(\bar{C})=r_{R}(C)=1$. Also, by Proposition 2.6(i), we have $\mathrm{G}_{\bar{C}}$ - $\operatorname{dim} \bar{R}(\bar{M})=\mathrm{G}_{C^{-}} \operatorname{dim}_{R}(M)<\infty$. Hence, by induction hypothesis, $\bar{C}$ is dualizing for $\bar{R}$, whence $C$ is dualizing for $R$ by Proposition 2.8(ii).

It is well-known that the existence of a finitely generated (resp. Cohen-Macaulay) module of finite injective (resp. projective) dimension implies Cohen-Macaulyness of the ring. But, in the special case that $C$ is dualizing, the proof is easy, as the following relations show

$$
\operatorname{dim}(R)=\operatorname{dim}_{R}(C) \leq \operatorname{id}_{R}(C)=\operatorname{depth}(R),
$$

where the first equality is from Proposition 2.4(i), and the remaining parts are from 2 , Theorem 3.1.17]. Therefore, in view of Theorem 3.4, we can state the following corollary.

Corollary 3.5. Let $(R, \mathfrak{m})$ be local. If there exists a Cohen-Macaulay R-module of type 1 and of finite $\mathrm{G}_{C^{-}}$dimension, then $R$ is Cohen-Macaulay.

\section{C-Injective modules}

In this section, our aim is to extend two nice results of J.Xu [22]. It is well-known that a Noetherian ring $R$ is Gorenstein if and only if $\mu^{i}(\mathfrak{p}, R)=\delta_{i, h t(\mathfrak{p})}$ (the Kronecker $\delta$ ). As a generalization, J.Xu [22, Theorem 2.1], showed that $R$ is Gorenstein if and only if for any $R$-module $F$, the necessary and sufficient condition for $F$ to be flat is that $\mu^{i}(\mathfrak{p}, F)=0$ for all $\mathfrak{p} \in \operatorname{Spec}(R)$ and all $i \neq \operatorname{ht}(\mathfrak{p})$. Next, in [22, Theorem 3.2], he proved a dual for this theorem. Indeed, he proved that $R$ is Gorenstein if and only if for any $R$-module $E$, the necessary and sufficient condition for $E$ to be injective is that $\pi_{i}(\mathfrak{p}, E)=0$ for all $\mathfrak{p} \in \operatorname{Spec}(R)$ and 
all $i \neq$ ht $(\mathfrak{p})$. In the present section, first we generalize the mentioned results. Next, we use our new results to determine the minimal flat resolution of some top local cohomology of a Cohen-Macaulay local rings and their torsion products.

Lemma 4.1. The followings are equivalent:

(i) $C$ is pointwise dualizing.

(ii) $C$-fd ${ }_{R}(E(R / \mathfrak{m}))=\mathrm{ht}(\mathfrak{m})$ for any $\mathfrak{m} \in \operatorname{Max}(R)$.

(iii) $C$-fd ${ }_{R}(E(R / \mathfrak{m}))<\infty$ for any $\mathfrak{m} \in \operatorname{Max}(R)$.

(iv) $C$-fd ${ }_{R}(E(R / \mathfrak{p}))=\mathrm{ht}(\mathfrak{p})$ for any $\mathfrak{p} \in \operatorname{Spec}(R)$.

(v) $C$-fd ${ }_{R}(E(R / \mathfrak{p}))<\infty$ for any $\mathfrak{p} \in \operatorname{Spec}(R)$.

(vi) $C$-id ${ }_{R}\left(T_{\mathfrak{m}}\right)=\mathrm{ht}(\mathfrak{m})$ for any $\mathfrak{m} \in \operatorname{Max}(R)$.

(vii) $C$-id $R_{R}\left(T_{\mathfrak{m}}\right)<\infty$ for any $\mathfrak{m} \in \operatorname{Max}(R)$.

(viii) $C$-id $R_{R}\left(T_{\mathfrak{p}}\right)=\mathrm{ht}(\mathfrak{p})$ for any $\mathfrak{p} \in \operatorname{Spec}(R)$.

(ix) $C$-id ${ }_{R}\left(T_{\mathfrak{p}}\right)<\infty$ for any $\mathfrak{p} \in \operatorname{Spec}(R)$.

Proof. (i) $\Longrightarrow$ (ii). Assume that $\mathfrak{m} \in \operatorname{Max}(R)$. There are equalities

$$
\begin{aligned}
C-\operatorname{fd}_{R}(E(R / \mathfrak{m})) & =\mathrm{fd}_{R}\left(\operatorname{Hom}_{R}(C, E(R / \mathfrak{m}))\right) \\
& =\operatorname{fd}_{R_{\mathfrak{m}}}\left(\operatorname{Hom}_{R_{\mathfrak{m}}}\left(C_{\mathfrak{m}}, E_{R_{\mathfrak{m}}}\left(R_{\mathfrak{m}} / \mathfrak{m} R_{\mathfrak{m}}\right)\right)\right. \\
& =\operatorname{id}_{R_{\mathfrak{m}}}\left(C_{\mathfrak{m}}\right) \\
& =\operatorname{dim}\left(R_{\mathfrak{m}}\right) \\
& =\operatorname{ht}(\mathfrak{m}),
\end{aligned}
$$

in which the first equality is from Theorem 2.9(ii), and the second one is from Remark 2.16.

(ii) $\Longrightarrow$ (iii). Is clear.

(iii) $\Longrightarrow($ i). We can assume that $(R, \mathfrak{m})$ is local. Now one can use Theorem 2.9(ii), to see that

$$
\begin{aligned}
\operatorname{id}_{R}(C) & =\operatorname{fd}_{R}\left(\operatorname{Hom}_{R}(C, E(R / \mathfrak{m}))\right) \\
& =C-\operatorname{fd}_{R}(E(R / \mathfrak{m}))<\infty,
\end{aligned}
$$

whence $C$ is dualizing.

(i) $\Longrightarrow$ (iv). Let $\mathfrak{p}$ be a prime ideal of $R$. Note that $E(R / \mathfrak{p})_{\mathfrak{q}} \neq 0$ if and only if $\mathfrak{q} \subseteq \mathfrak{p}$. Now as in (i) $\Longrightarrow\left(\right.$ ii), we have $C$-fd ${ }_{R}(E(R / \mathfrak{p}))=\operatorname{dim}\left(R_{\mathfrak{p}}\right)=\mathrm{ht}(\mathfrak{p})$.

(iv) $\Longrightarrow(\mathrm{v})$. Is clear.

(v) $\Longrightarrow$ (i). Again, we can assume that $R$ is local. Now the proof is similar to that of (iii) $\Longrightarrow($ i).

(ii) $\Longleftrightarrow$ (vi) and (iii) $\Longleftrightarrow\left(\right.$ vii). Note that $T_{\mathfrak{m}}=\operatorname{Hom}_{R}\left(E(R / \mathfrak{m}), E(R / \mathfrak{m})^{(X)}\right)$ for some set $X$. Now we have the equalities

$$
\begin{aligned}
C-\operatorname{id}_{R}\left(T_{\mathfrak{m}}\right) & =\operatorname{id}_{R}\left(C \otimes_{R} T_{\mathfrak{m}}\right) \\
& =\operatorname{id}_{R}\left(C \otimes_{R} \operatorname{Hom}_{R}\left(E(R / \mathfrak{m}), E(R / \mathfrak{m})^{(X)}\right)\right) \\
& =\operatorname{id}_{R}\left(\operatorname{Hom}_{R}\left(\operatorname{Hom}_{R}(C, E(R / \mathfrak{m})), E(R / \mathfrak{m})^{(X)}\right)\right) \\
& =\operatorname{fd}_{R}\left(\operatorname{Hom}_{R}(C, E(R / \mathfrak{m}))\right) \\
& =C-\operatorname{fd}_{R}(E(R / \mathfrak{m})),
\end{aligned}
$$


in which the first equality is from Theorem 2.9(i), the fourth equality is from Remark 2.16 and the fact that $E(R / \mathfrak{m})^{(X)}$ is an injective cogenerator in the category of $R_{\mathfrak{m}}$-modules, and the last one is from Theorem 2.9(ii).

(iv) $\Longleftrightarrow$ (viii) and (v) $\Longleftrightarrow(\mathrm{ix})$. Are similar to (ii) $\Longleftrightarrow(\mathrm{vi})$.

The following theorem is a generalization of [21, theorem 2.1].

Theorem 4.2. The following are equivalent:

(i) $C$ is pointwise dualizing.

(ii) An R-module $M$ is $C$-flat if and only if $\mu^{i}(\mathfrak{p}, M)=0$ for all $\mathfrak{p} \in \operatorname{Spec}(R)$ whenever $i \neq$ ht $(\mathfrak{p})$.

(iii) An $R$-module $M$ is flat if and only if $\mu^{i}\left(\mathfrak{p}, C \otimes_{R} M\right)=0$ for all $\mathfrak{p} \in \operatorname{Spec}(R)$ whenever $i \neq$ ht $(\mathfrak{p})$.

Proof. (i) $\Longrightarrow\left(\right.$ ii). First assume that $M$ is $C$-flat. Set $M=C \otimes_{R} F$, where $F$ is a flat $R$-module. Since $C$ is pointwise dualizing, we have $\mu^{i}(\mathfrak{p}, C)=0$ for all $\mathfrak{p} \in \operatorname{Spec}(R)$ with $i \neq \mathrm{ht}(\mathfrak{p})$. Assume that

$$
0 \rightarrow C \rightarrow E^{0}(C) \rightarrow E^{1}(C) \rightarrow \ldots \rightarrow E^{i}(C) \rightarrow \ldots
$$

is the minimal injective resolution of $C$. By applying the exact functor $-\otimes_{R} F$ to this resolution, we find an exact complex

$$
0 \rightarrow M=C \otimes_{R} F \rightarrow E^{0}(C) \otimes_{R} F \rightarrow E^{1}(C) \otimes_{R} F \rightarrow \ldots \rightarrow E^{i}(C) \otimes_{R} F \rightarrow \ldots,(*)
$$

which is an injective resolution for $M$. By [7, Theorem 3.3.12] the injective $R$-module $E(R / \mathfrak{p}) \otimes_{R} F$ is a direct sum of copies of $E(R / \mathfrak{p})$ for each $\mathfrak{p} \in \operatorname{Spec}(R)$. Now, since the minimal injective resolution of $M$ is a direct summand of the complex $(*)$, we get the result. Conversely, suppose that $M$ is an $R$-module such that $\mu^{i}(\mathfrak{p}, M)=0$ for all $\mathfrak{p} \in \operatorname{Spec}(R)$ whenever $i \neq \mathrm{ht}(\mathfrak{p})$. In order to show that $M$ is $C$-flat, it is enough to prove that $M_{\mathfrak{m}}$ is $C_{\mathfrak{m}}$ flat $R_{\mathfrak{m}}$-module for all $\mathfrak{m} \in \operatorname{Max}(R)$. For if $M_{\mathfrak{m}}$ is $C_{\mathfrak{m}}$-flat $R_{\mathfrak{m}}$-module for all $\mathfrak{m} \in \operatorname{Max}(R)$, then $\operatorname{Hom}_{R}(C, M)_{\mathfrak{m}} \cong \operatorname{Hom}_{R_{\mathfrak{m}}}\left(C_{\mathfrak{m}}, M_{\mathfrak{m}}\right)$ is flat as an $R_{\mathfrak{m}}$-module for all $\mathfrak{m} \in \operatorname{Max}(R)$ by Theorem 2.9(ii). Hence $\operatorname{Hom}_{R}(C, M)$ is a flat $R$-module and thus $M$ is $C$-flat by Theorem 2.9 (ii). Hence, replacing $R$ by $R_{\mathfrak{m}}$, we can assume that $(R, \mathfrak{m})$ is local. Clearly we may assume that $M \neq 0$. In this case we have id $R_{R}(M)<\infty$ since by assumption $\mu^{i}(\mathfrak{p}, M)=0$ for all $\mathfrak{p} \in \operatorname{Spec}(R)$ and all $i>\operatorname{dim}(R)$. Hence the assumption in conjunction with Lemma 4.1, imply that $M$ has a bounded injective resolution all of whose terms have finite $C$-flat dimensions. More precisely, by Lemma 4.1, if $E^{i}$ is the $i$-th term in the minimal injective resolution of $M$, then $C-\mathrm{fd}_{R}\left(E^{i}\right)=i$ for all $0 \leq i \leq \mathrm{id}(M)$. Breaking up this resolution to short exact sequences and using [19, Corollary 5.7], we can conclude that $C-\operatorname{fd}_{R}(M)=0$. Hence $M$ is $C$-flat, as wanted.

(ii) $\Longrightarrow$ (iii). Assume that $M$ is a flat $R$-module. Then $C \otimes_{R} M \in \mathcal{F}_{C}$ and $\mu^{i}\left(\mathfrak{p}, C \otimes_{R}\right.$ $M)=0$ for all $\mathfrak{p} \in \operatorname{Spec}(R)$ whenever $i \neq \mathrm{ht}(\mathfrak{p})$ by assumption. Conversely, suppose that $\mu^{i}\left(\mathfrak{p}, C \otimes_{R} M\right)=0$ for all $\mathfrak{p} \in \operatorname{Spec}(R)$ whenever $i \neq \mathrm{ht}(\mathfrak{p})$. Then, by assumption, $C \otimes_{R} M$ is $C$-flat. Set $C \otimes_{R} M=C \otimes_{R} F$, where $F$ is flat. Therefore $C \otimes_{R} M \in \mathcal{B}_{C}(R)$, whence 
$M \in \mathcal{A}_{C}(R)$ by [18, Theorem 2.8(b)]. Thus we have the isomorphisms

$$
\begin{aligned}
M & \cong \operatorname{Hom}_{R}\left(C, C \otimes_{R} M\right) \\
& \cong \operatorname{Hom}_{R}\left(C, C \otimes_{R} F\right) \\
& \cong F,
\end{aligned}
$$

where the first and the last isomorphism hold since both $M$ and $F$ are in $\mathcal{A}_{C}(R)$.

(iii) $\Longrightarrow$ (i). Note that $R$ is a flat $R$-module. Hence by assumption, if $\mathfrak{m} \in \operatorname{Max}(R)$, then $\mu^{i}\left(\mathfrak{m}, C \otimes_{R} R\right)=0$ for all $i>$ ht $(\mathfrak{m})$. Thus id $R_{\mathfrak{m}}\left(C_{\mathfrak{m}}\right)<\infty$, as wanted.

Theorem 4.3. The following are equivalent:

(i) $C$ is pointwise dualizing.

(ii) An R-module $M$ is $C$-injective if and only if $\pi_{i}(\mathfrak{p}, M)=0$ for all $\mathfrak{p} \in \operatorname{Spec}(R)$ whenever $i \neq h t(\mathfrak{p})$.

(iii) An R-module $M$ is injective if and only if $\pi_{i}\left(\mathfrak{p}, \operatorname{Hom}_{R}(C, M)\right)=0$ for all $\mathfrak{p} \in$ $\operatorname{Spec}(R)$ whenever $i \neq h t(\mathfrak{p})$.

Proof. (i) $\Longrightarrow$ (ii). Assume that $M$ is a nonzero $C$-injective $R$-module. Set $M=\operatorname{Hom}_{R}(C, E)$ with $E$ is injective. First, we show that $M$ is cotorsion. Assume that $F$ is a flat $R$-module. Then, by [7. Theorem 3.2.1], we have $\operatorname{Ext}_{R}^{1}\left(F, \operatorname{Hom}_{R}(C, E)\right) \cong \operatorname{Hom}_{R}\left(\operatorname{Tor}_{1}^{R}(F, C), E\right)=0$, and hence $M$ is cotorsion. Fix a prime ideal $\mathfrak{p}$ of $R$ and set $k(\mathfrak{p})=R_{\mathfrak{p}} / \mathfrak{p} R_{\mathfrak{p}}$. Note that $\operatorname{Hom}_{R}\left(R_{\mathfrak{p}}, E\right)$ is an injective $R$-module and that $\operatorname{Hom}_{R}\left(R_{\mathfrak{p}}, E\right) \cong \underset{\mathfrak{q} \in X}{\oplus} E(R / \mathfrak{q})$, where $X \subseteq \operatorname{Ass}_{R}(E)$ and each element of $X$ is a subset of $\mathfrak{p}$. There are isomorphisms

$$
\begin{aligned}
\operatorname{Tor}_{i}^{R_{\mathfrak{p}}}\left(k(\mathfrak{p}), \operatorname{Hom}_{R}\left(R_{\mathfrak{p}}, \operatorname{Hom}_{R}(C, E)\right)\right) & \cong \operatorname{Tor}_{i}^{R_{\mathfrak{p}}}\left(k(\mathfrak{p}), \operatorname{Hom}_{R}\left(C_{\mathfrak{p}}, E\right)\right) \\
& \cong \operatorname{Tor}^{R_{\mathfrak{p}}}\left(k(\mathfrak{p}), \operatorname{Hom}_{R_{\mathfrak{p}}}\left(C_{\mathfrak{p}}, \operatorname{Hom}_{R}\left(R_{\mathfrak{p}}, E\right)\right)\right. \\
& \cong \operatorname{Tor}_{i}^{R_{\mathfrak{p}}}\left(k(\mathfrak{p}), \operatorname{Hom}_{R_{\mathfrak{p}}}\left(C_{\mathfrak{p}}, \underset{\mathfrak{q} \in X}{\oplus} E(R / \mathfrak{q})\right)\right. \\
& \cong \operatorname{Hom}_{R_{\mathfrak{p}}}\left(\operatorname{Ext}_{R_{\mathfrak{p}}}^{i}\left(k(\mathfrak{p}), C_{\mathfrak{p}}\right), \underset{\mathfrak{q} \in X}{\oplus} E(R / \mathfrak{q})\right),
\end{aligned}
$$

where the last isomorphism is from [7, Theorem 3.2.13]. Now since $C_{\mathfrak{p}}$ is dualizing for $R_{\mathfrak{p}}$, we have $\operatorname{Ext}_{R_{\mathfrak{p}}}^{i}\left(k(\mathfrak{p}), C_{\mathfrak{p}}\right)=0$ for all $i \neq \mathrm{ht}(\mathfrak{p})$. Therefore $\pi_{i}(\mathfrak{p}, M)=0$ for all $i \neq \mathrm{ht}(\mathfrak{p})$. Conversely, assume that $M$ is a non-zero $R$-module with $\pi_{i}(\mathfrak{p}, M)=0$ for all $i \neq \mathrm{ht}(\mathfrak{p})$. By assumption, the minimal flat resolution of $M$ is of the form

$$
\cdots \longrightarrow F_{i} \longrightarrow \cdots \longrightarrow F_{1} \longrightarrow F_{0} \longrightarrow M \longrightarrow 0,
$$

in which $F_{i}=\prod_{\operatorname{ht}(\mathfrak{p})=i} T_{\mathfrak{p}}$ for all $i \geq 1$. Also, in view of [22, Lemma 3.1], we have $F_{0}=\prod_{h t(\mathfrak{p})=0} T_{\mathfrak{p}}$. Hence the minimal flat resolution of $M$ is of the form

$$
\cdots \longrightarrow \prod_{\operatorname{ht}(\mathfrak{p})=i} T_{\mathfrak{p}} \longrightarrow \cdots \longrightarrow \prod_{\mathrm{ht}(\mathfrak{p})=1} T_{\mathfrak{p}} \longrightarrow \prod_{\mathrm{ht}(\mathfrak{p})=0} T_{\mathfrak{p}} \longrightarrow M \longrightarrow 0 .(*)
$$

Let $E$ be an injective cogenerator. According to Lemma 2.10(i), it is enough to show that $\operatorname{Hom}_{R}(M, E)$ is $C$-flat. In fact, by Theorem 4.2, we need only to show that $\mu^{i}\left(\mathfrak{p}, \operatorname{Hom}_{R}(M, E)\right)=0$ for all $i \neq \mathrm{ht}(\mathfrak{p})$ and all $i \geq 0$. Applying the exact functor $\operatorname{Hom}_{R}(-, E)$ on $(*)$, we get an injective resolution

$$
0 \longrightarrow \operatorname{Hom}_{R}(M, E) \longrightarrow \operatorname{Hom}_{R}\left(\prod_{\mathrm{ht}(\mathfrak{p})=0} T_{\mathfrak{p}}, E\right) \longrightarrow
$$




$$
\operatorname{Hom}_{R}\left(\prod_{\mathrm{ht}(\mathfrak{p})=1} T_{\mathfrak{p}}, E\right) \longrightarrow \cdots \longrightarrow \operatorname{Hom}_{R}\left(\prod_{\mathrm{ht}(\mathfrak{p})=i} T_{\mathfrak{p}}, E\right) \longrightarrow \cdots
$$

for $\operatorname{Hom}_{R}(M, E)$. Note that $\operatorname{Hom}_{R}\left(\prod_{\mathrm{ht}(\mathfrak{p})=i} T_{\mathfrak{p}}, E\right)$ is an injective $R$-module for all $i \geq 0$. Set $\operatorname{Hom}_{R}\left(\prod_{\operatorname{ht}(\mathfrak{p})=i} T_{\mathfrak{p}}, E\right) \cong \oplus E(R / \mathfrak{q})$. We show that ht $(\mathfrak{q})=i$. Since $C$ is pointwise dualizing, by Lemma 4.1 , we have $C-\mathrm{fd}_{R}(E(R / \mathfrak{q}))=\mathrm{ht}(\mathfrak{q})$. On the other hand, we have the equalities

$$
\begin{aligned}
C-\mathrm{fd}_{R}(E(R / \mathfrak{q})) & =C-\mathrm{fd}_{R}(\oplus E(R / \mathfrak{q})) \\
& =C-\mathrm{fd}_{R}\left(\operatorname{Hom}_{R}\left(\prod_{\mathrm{ht}(\mathfrak{p})=i} T_{\mathfrak{p}}, E\right)\right) \\
& =C-\mathrm{id}_{R}\left(\prod_{\operatorname{ht}(\mathfrak{p})=i} T_{\mathfrak{p}}\right) \\
& =i,
\end{aligned}
$$

in which the third equality is from Lemma 2.10(i), and the last one is from Lemma 4.1. Hence $\mu^{i}\left(\mathfrak{p}, \operatorname{Hom}_{R}(M, E)\right)=0$ for all $i \geq 0$ with $i \neq \mathrm{ht}(\mathfrak{p})$, as wanted.

(ii) $\Longrightarrow$ (iii). Assume that $M$ is an injective $R$-module. Then $\operatorname{Hom}_{R}(C, M) \in \mathcal{I}_{C}$ and $\mu^{i}\left(\mathfrak{p}, \operatorname{Hom}_{R}(C, M)\right)=0$ for all $\mathfrak{p} \in \operatorname{Spec}(R)$ whenever $i \neq \mathrm{ht}(\mathfrak{p})$ by assumption. Conversely, suppose that $\mu^{i}\left(\mathfrak{p}, \operatorname{Hom}_{R}(C, M)\right)=0$ for all $\mathfrak{p} \in \operatorname{Spec}(R)$ whenever $i \neq \mathrm{ht}(\mathfrak{p})$. Then, by assumption, $\operatorname{Hom}_{R}(C, M)$ is $C$-injective. Set $\operatorname{Hom}_{R}(C, M)=\operatorname{Hom}_{R}(C, I)$, where $I$ is injective. Therefore $\operatorname{Hom}_{R}(C, M) \in \mathcal{A}_{C}(R)$, whence $M \in \mathcal{B}_{C}(R)$ by [18, Theorem 2.8(a)]. Thus we have the isomorphisms

$$
\begin{aligned}
M & \cong C \otimes_{R} \operatorname{Hom}_{R}(C, M) \\
& \cong C \otimes_{R} \operatorname{Hom}_{R}(C, I) \\
& \cong I
\end{aligned}
$$

where the first and the last isomorphism hold since both $M$ and $I$ are in $\mathcal{B}_{C}(R)$.

(iii) $\Longrightarrow$ (i). Assume that $\mathfrak{m}$ is a maximal ideal of $R$. Set $k(\mathfrak{m})=R_{\mathfrak{m}} / \mathfrak{m} R_{\mathfrak{m}}$. Since $E(R / \mathfrak{m})$ is injective, by assumption, we have $\pi_{i}\left(\mathfrak{m}, \operatorname{Hom}_{R}(C, E(R / \mathfrak{m}))\right)=0$ fo all $i \neq \mathrm{ht}(\mathfrak{m})$. On the other hand, there are isomorphisms

$$
\begin{aligned}
\operatorname{Hom}_{R_{\mathfrak{m}}}\left(\operatorname{Ext}_{R_{\mathfrak{m}}}^{i}\left(k(\mathfrak{m}), C_{\mathfrak{m}}\right), E(k(\mathfrak{m}))\right) & \cong \operatorname{Tor}_{i}^{R_{\mathfrak{m}}}\left(k(\mathfrak{m}), \operatorname{Hom}_{R_{\mathfrak{m}}}\left(C_{\mathfrak{m}}, E(k(\mathfrak{m}))\right)\right. \\
& \cong \operatorname{Tor}_{i}^{R_{\mathfrak{m}}}\left(k(\mathfrak{m}), \operatorname{Hom}_{R_{\mathfrak{m}}}\left(C_{\mathfrak{m}} \otimes_{R_{\mathfrak{m}}} R_{\mathfrak{m}}, E(k(\mathfrak{m}))\right)\right. \\
& \cong \operatorname{Tor}_{i}^{R_{\mathfrak{m}}}\left(k(\mathfrak{m}), \operatorname{Hom}_{R}\left(R_{\mathfrak{m}}, \operatorname{Hom}_{R_{\mathfrak{m}}}\left(C_{\mathfrak{m}}, E(k(\mathfrak{m}))\right)\right.\right. \\
& \cong \operatorname{Tor}_{i}^{R_{\mathfrak{m}}}\left(k(\mathfrak{m}), \operatorname{Hom}_{R}\left(R_{\mathfrak{m}}, \operatorname{Hom}_{R}(C, E(R / \mathfrak{m}))\right),\right.
\end{aligned}
$$

where the first isomorphism is from [7, Theorem 3.2.13], and the last one is from Remark 2.16. From this isomorphisms, it follows that $\operatorname{Hom}_{R_{\mathfrak{m}}}\left(\operatorname{Ext}_{R_{\mathfrak{m}}}^{i}\left(k(\mathfrak{m}), C_{\mathfrak{m}}\right), E(k(\mathfrak{m}))\right)=0$ for all $i \neq \mathrm{ht}(\mathfrak{m})$, from which we conclude that $\operatorname{Ext}_{R_{\mathfrak{m}}}^{i}\left(k(\mathfrak{m}), C_{\mathfrak{m}}\right)=0$ for all $i \neq \mathrm{ht}(\mathfrak{m})$, since $E(k(\mathfrak{m}))$ is an injective cogenerator in the category of $R_{\mathfrak{m}}$-modules. Thus $C_{\mathfrak{m}}$ is dualizing for $R_{\mathfrak{m}}$, as required.

Corollary 4.4. Let $C$ be pointwise dualizing. Then flat cover of any $C$-injective $R$-module is C-injective. 
Proof. By Lemma 4.1, $C$-id ${ }_{R}\left(T_{\mathfrak{p}}\right)=0$ for any prime ideal $\mathfrak{p}$ with ht $(\mathfrak{p})=0$. Hence $T_{\mathfrak{p}}$ is $C$-injective for any prime ideal $\mathfrak{p}$ with ht $(\mathfrak{p})=0$. Assume that $M$ is a $C$-injective $R$-module. By Theorem 4.3, we have $F(M)=\prod_{\text {ht }(\mathfrak{p})=0} T_{\mathfrak{p}}$. Now the result follows since the class $\mathcal{I}_{C}$ closed under arbitrary direct product.

Corollary 4.5. The $R$-module $C$ is pointwise dualizing if and only if for any prime ideal $\mathfrak{p}$ of $R$,

$$
\pi_{i}\left(\mathfrak{p}, \operatorname{Hom}_{R}(C, E(R / \mathfrak{p}))\right)= \begin{cases}1 & i=\mathrm{ht}(\mathfrak{p}), \\ 0 & i \neq \mathrm{ht}(\mathfrak{p}) .\end{cases}
$$

Proof. Assume that $\mathfrak{p} \in \operatorname{Spec}(R)$. Set $k(\mathfrak{p})=R_{\mathfrak{p}} / \mathfrak{p} R_{\mathfrak{p}}$. We have the following equalities

$$
\begin{aligned}
\pi_{i}\left(\mathfrak{p}, \operatorname{Hom}_{R}(C, E(R / \mathfrak{p}))\right) & =\operatorname{vdim}_{k(\mathfrak{p})} \operatorname{Tor}_{i}^{R_{\mathfrak{p}}}\left(k(\mathfrak{p}), \operatorname{Hom}_{R}\left(R_{\mathfrak{p}}, \operatorname{Hom}_{R}(C, E(R / \mathfrak{p}))\right)\right. \\
& =\operatorname{vdim}_{k(\mathfrak{p})} \operatorname{Tor}_{i}^{R_{\mathfrak{p}}}\left(k(\mathfrak{p}), \operatorname{Hom}_{R}\left(C_{\mathfrak{p}}, \operatorname{Hom}_{R_{\mathfrak{p}}}\left(R_{\mathfrak{p}}, E(R / \mathfrak{p})\right)\right)\right. \\
& =\operatorname{vdim}_{k(\mathfrak{p})} \operatorname{Tor}_{i}^{R_{\mathfrak{p}}}\left(k(\mathfrak{p}), \operatorname{Hom}_{R}\left(C_{\mathfrak{p}}, E(R / \mathfrak{p})\right)\right) \\
& =\operatorname{vdim}_{k(\mathfrak{p})} \operatorname{Hom}_{R_{\mathfrak{p}}}\left(\operatorname{Ext}_{R_{\mathfrak{p}}}^{i}\left(k(\mathfrak{p}), C_{\mathfrak{p}}\right), E(R / \mathfrak{p})\right),
\end{aligned}
$$

where the second equality is from Remark 2.16, and the last equality is from [7, Theorem 3.2.13]. Now, $C$ is pointwise dualizing if and only if $C_{\mathfrak{p}}$ is the dualizing module of $R_{\mathfrak{p}}$ for all $\mathfrak{p} \in \operatorname{Spec}(R)$, and this is the case if and only if

$$
\operatorname{Ext}_{R_{\mathfrak{p}}}^{i}\left(k(\mathfrak{p}), C_{\mathfrak{p}}\right) \cong\left\{\begin{array}{cl}
k(\mathfrak{p}) & i=\mathrm{ht}(\mathfrak{p}), \\
0 & i \neq \mathrm{ht}(\mathfrak{p}) .
\end{array}\right.
$$

for all $\mathfrak{p} \in \operatorname{Spec}(R)$. Thus we are done by the above equalities and the fact that $\operatorname{Hom}_{R_{\mathfrak{p}}}(k(\mathfrak{p}), E(R / \mathfrak{p})) \cong k(\mathfrak{p})$.

In the following corollaries, we are concerned with the local cohomology. For an $R$ module $M$, the $i$-th local cohomology module of $M$ with respect to an ideal $\mathfrak{a}$ of $R$, denoted by $\mathrm{H}_{\mathfrak{a}}^{i}(M)$, is defined to be

$$
\mathrm{H}_{\mathfrak{a}}^{i}(M)=\underset{n \geq 1}{\lim \operatorname{Ext}_{R}^{i}\left(R / \mathfrak{a}^{n}, M\right)}
$$

For the basic properties of local cohomology modules, please see the textbook [3].

Corollary 4.6. Let $(R, \mathfrak{m})$ be a Cohen-Macaulay local ring with $\operatorname{dim}(R)=d$ possesing a canonical module $\omega_{R}$. Then $\pi_{i}\left(\mathfrak{m}, \mathrm{H}_{\mathfrak{m}}^{d}(R)\right)=\delta_{i, d}$, and $\pi_{i}\left(\mathfrak{q}, \mathrm{H}_{\mathfrak{m}}^{d}(R)\right)=0$ for any nonmaximal prime ideal $\mathfrak{q}$ whenever $i \neq \mathrm{ht}(\mathfrak{q})$.

Proof. By [3, Theorem 11.2.8], we have $\mathrm{H}_{\mathfrak{m}}^{d}(R) \cong \operatorname{Hom}_{R}\left(\omega_{R}, E(R / \mathfrak{m})\right)$, and hence $\mathrm{H}_{\mathfrak{m}}^{d}(R)$ is $\omega_{R}$-injective. Assume that $\mathfrak{q}$ is a non-maximal prime ideal of $R$. Then by the Theorem 4.3, we have $\pi_{i}\left(\mathfrak{q}, \mathrm{H}_{\mathfrak{m}}^{d}(R)\right)=0$ for all $i \neq \mathrm{ht}(\mathfrak{q})$. Finally, by corollary 4.5 , we have $\pi_{i}\left(\mathfrak{m}, \mathrm{H}_{\mathfrak{m}}^{d}(R)\right)=0$ for all $i \neq d$ and that $\pi_{d}\left(\mathfrak{m}, \mathrm{H}_{\mathfrak{m}}^{d}(R)\right)=1$, as wanted.

If $(R, \mathfrak{m})$ is a Cohen-Macaulay local ring with $\operatorname{dim}(R)=d$, then by [3, Corollary 6.2.9] the only non-vanishing local cohomology of $R$ with respect to $\mathfrak{m}$ is $\mathrm{H}_{\mathfrak{m}}^{d}(R)$. Also, if $R$ admits a 
canonical module, then by [7, Proposition 9.5.22], we have $\mathrm{fd}_{R}\left(\mathrm{H}_{\mathfrak{m}}^{d}(R)\right)=d$. The following corollary describes the structure of the minimal flat resolution of $\mathrm{H}_{\mathfrak{m}}^{d}(R)$.

Corollary 4.7. Let $(R, \mathfrak{m})$ be a d-dimensional Cohen-Macaulay local ring possessing a canonical module. The minimal flat resolution of $\mathrm{H}_{\mathfrak{m}}^{d}(R)$ is of the form

$$
0 \longrightarrow \widehat{R_{\mathfrak{m}}} \longrightarrow \cdots \longrightarrow \prod_{\text {ht }(\mathfrak{p})=1} T_{\mathfrak{p}} \longrightarrow \prod_{\mathrm{ht}(\mathfrak{p})=0} T_{\mathfrak{p}} \longrightarrow \mathrm{H}_{\mathfrak{m}}^{d}(R) \longrightarrow 0 .
$$

In the following corollary, we give another proof of [16, Corollary 3.7]. Our approach is direct, and uses the well-known fact that the homology functor Tor can be computed by a flat resolution.

Corollary 4.8. Let $(R, \mathfrak{m})$ be a d-dimensional Cohen-Macaulay local ring. Then

$$
\operatorname{Tor}_{i}^{R}\left(\mathrm{H}_{\mathfrak{m}}^{d}(R), \mathrm{H}_{\mathfrak{m}}^{d}(R)\right) \cong\left\{\begin{array}{cl}
\mathrm{H}_{\mathfrak{m}}^{d}(R) & i=d, \\
0 & i \neq d .
\end{array}\right.
$$

Proof. Note that $\widehat{R}$ is a $d$-dimensional complete Cohen-Macaulay local ring, and hence admits a canonical module $\omega_{\widehat{R}}$. The $R$-module $\mathrm{H}_{\mathfrak{m}}^{d}(R)$ is Artinian by [3, Theorem 7.1.6], and thus naturally has a $\widehat{R}$-module structure by [3, Remark 10.2.9]. Hence $\operatorname{Tor}_{i}^{R}\left(\mathrm{H}_{\mathfrak{m}}^{d}(R), \mathrm{H}_{\mathfrak{m}}^{d}(R)\right)$ is Artinian for all $i \geq 0$ by [14, Corollary 3.2]. Thus there are isomorphisms

$$
\begin{aligned}
\operatorname{Tor}_{i}^{R}\left(\mathrm{H}_{\mathfrak{m}}^{d}(R), \mathrm{H}_{\mathfrak{m}}^{d}(R)\right) & \cong \operatorname{Tor}_{i}^{R}\left(\mathrm{H}_{\mathfrak{m}}^{d}(R), \mathrm{H}_{\mathfrak{m}}^{d}(R)\right) \otimes_{R} \widehat{R} \\
& \cong \operatorname{Tor}_{i}^{\widehat{R}}\left(\mathrm{H}_{\mathfrak{m}}^{d}(R) \otimes_{R} \widehat{R}, \mathrm{H}_{\mathfrak{m}}^{d}(R) \otimes_{R} \widehat{R}\right) \\
& \cong \operatorname{Tor}_{i} \widehat{R}\left(\mathrm{H}_{\mathfrak{m} \widehat{R}}^{d}(\widehat{R}), \mathrm{H}_{\mathfrak{m} \widehat{R}}^{d}(\widehat{R})\right),
\end{aligned}
$$

in which the second isomorphism is from [7, Theorem 2.1.11], and the last one is flat base change [3, Theorem 4.3.2]. Also, we have the isomorphisms

$$
\begin{aligned}
\mathrm{H}_{\mathfrak{m}}^{d}(R) & \cong \mathrm{H}_{\mathfrak{m}}^{d}(R) \otimes_{R} \widehat{R} \\
& \cong \mathrm{H}_{\mathfrak{m} \widehat{R}}^{d}(\widehat{R}) \\
& \cong \operatorname{Hom}_{\widehat{R}}\left(\omega_{\widehat{R}}, E_{\widehat{R}}(\widehat{R} / \mathfrak{m} \widehat{R})\right)
\end{aligned}
$$

in which the first isomorphism holds because $\mathrm{H}_{\mathfrak{m}}^{d}(R)$ is Artinian, the second isomorphism is the flat base change, and the last one is local duality [3. Theorem 11.2.8]. Thus $\mathrm{H}_{\mathfrak{m}}^{d}(R)$ is a $\omega_{\widehat{R}}$-injective $\widehat{R}$-module. Hence, by Corollary 4.7 , the minimal flat resolution of $\mathrm{H}_{\mathfrak{m}}^{d}(R)$, as an $\widehat{R}$-module, is of the form

$$
0 \longrightarrow \widehat{R}_{\mathfrak{m} \widehat{R}} \longrightarrow \cdots \longrightarrow \prod_{\operatorname{ht}(Q)=1} T_{Q} \longrightarrow \prod_{\operatorname{ht}(Q)=0} T_{Q} \longrightarrow \mathrm{H}_{\mathfrak{m}}^{d}(R) \longrightarrow 0
$$

in which $T_{Q}$ is the completion of a free $\widehat{R}_{Q}$-module with respect to $Q \widehat{R}_{Q}$-adic topology, for $Q \in \operatorname{Spec}(\widehat{R})$. Observe that the above resolution is a flat resolution of $\mathrm{H}_{\mathfrak{m}}^{d}(R)$ as an $R$-module since the modules in the above resolution are all flat $R$-modules. Therefore, we can replace $R$ by $\widehat{R}$, and assume that $R$ is complete. So that, the minimal flat resolution of $\mathrm{H}_{\mathfrak{m}}^{d}(R)$ is of the form

$$
0 \longrightarrow \widehat{R_{\mathfrak{m}}} \longrightarrow \cdots \longrightarrow \prod_{h t(\mathfrak{p})=1} T_{\mathfrak{p}} \longrightarrow \prod_{h t(\mathfrak{p})=0} T_{\mathfrak{p}} \longrightarrow \mathrm{H}_{\mathfrak{m}}^{d}(R) \longrightarrow 0
$$


in which $T_{\mathfrak{p}}$ is the completion of a free $R_{\mathfrak{p}}$-module with respect to $\mathfrak{p} R_{\mathfrak{p}}$-adic topology, for $\mathfrak{p} \in \operatorname{Spec}(R)$. Next, note that for each prime ideal $\mathfrak{p}$ with $\mathfrak{p} \neq \mathfrak{m}$, we have $\mathrm{H}_{\mathfrak{m}}^{d}(R) \otimes_{R}\left(\prod T_{\mathfrak{p}}\right)=0$. Indeed, we can write $\mathrm{H}_{\mathfrak{m}}^{d}(R)=\underset{\alpha \in I}{\lim } M_{\alpha}$, where $M_{\alpha}$ is a finitely generated submodule of $\mathrm{H}_{\mathfrak{m}}^{d}(R)$. Also $T_{\mathfrak{p}}=\operatorname{Hom}_{R}\left(E(R / \mathfrak{p}), E(R / \mathfrak{p})^{(X)}\right)$ for some set $X$. Now since $M_{\alpha}$ is of finite length by [3, Theorem 7.1.3], we can take an element $x \in \mathfrak{m} \backslash \mathfrak{q}$ such that $x M_{\alpha}=0$. But multiplication of $x$ induces an automorphism on $E(R / \mathfrak{p})$ and hence on $\prod T_{\mathfrak{p}}$. Consequently, multiplication of $x$ on $M_{\alpha} \otimes_{R}\left(\prod T_{\mathfrak{p}}\right)$ is both an isomorphism and zero. Hence $M_{\alpha} \otimes_{R}\left(\prod T_{\mathfrak{p}}\right)=0$, from which we conclude that $\mathrm{H}_{\mathfrak{m}}^{d}(R) \otimes_{R}\left(\prod T_{\mathfrak{p}}\right)=0$ since tensor commutes with direct limit. Thus $\operatorname{Tor}_{i}^{R}\left(\mathrm{H}_{\mathfrak{m}}^{d}(R), \mathrm{H}_{\mathfrak{m}}^{d}(R)\right)=0$ for $i \neq d$. Finally, we have

$$
\begin{aligned}
\operatorname{Tor}_{d}^{R}\left(\mathrm{H}_{\mathfrak{m}}^{d}(R), \mathrm{H}_{\mathfrak{m}}^{d}(R)\right) & \cong \widehat{R_{\mathfrak{m}}} \otimes_{R} \mathrm{H}_{\mathfrak{m}}^{d}(R) \\
& \cong \mathrm{H}_{\mathfrak{m} \widehat{R_{\mathfrak{m}}}}^{d}\left(\widehat{R_{\mathfrak{m}}}\right) \\
& \cong \operatorname{Hom}_{\widehat{R_{\mathfrak{m}}}}\left(\widehat{\omega_{R_{\mathfrak{m}}}}, E_{\widehat{R_{\mathfrak{m}}}}\left(\widehat{R_{\mathfrak{m}}} / \mathfrak{m} \widehat{R_{\mathfrak{m}}}\right)\right) \\
& \cong \operatorname{Hom}_{R_{\mathfrak{m}}}\left(\omega_{R_{\mathfrak{m}}}, E_{R_{\mathfrak{m}}}\left(R_{\mathfrak{m}} / \mathfrak{m} R_{\mathfrak{m}}\right)\right) \otimes_{R_{\mathfrak{m}}} \widehat{R_{\mathfrak{m}}} \\
& \cong \operatorname{Hom}_{R_{\mathfrak{m}}}\left(\omega_{R_{\mathfrak{m}}}, E_{R_{\mathfrak{m}}}\left(R_{\mathfrak{m}} / \mathfrak{m} R_{\mathfrak{m}}\right)\right) \\
& \cong \operatorname{Hom}_{R}\left(\omega_{R}, E(R / \mathfrak{m})\right) \otimes_{R} R_{\mathfrak{m}} \\
& \cong \operatorname{Hom}_{R}\left(\omega_{R}, E(R / \mathfrak{m}) \otimes_{R} R_{\mathfrak{m}}\right) \\
& \cong \operatorname{Hom}_{R}\left(\omega_{R}, E(R / \mathfrak{m})\right) \\
& \cong \operatorname{H}_{\mathfrak{m}}^{d}(R)
\end{aligned}
$$

in which the second isomorphism is the flat base change [3, Theorem 4.3.2], the third isomorphism is local duality [3. Theorem 11.2.8], and the fifth one is from [3, Remark 10.2.9], since $\operatorname{Hom}_{R_{\mathfrak{m}}}\left(\omega_{R_{\mathfrak{m}}}, E_{R_{\mathfrak{m}}}\left(R_{\mathfrak{m}} / \mathfrak{m} R_{\mathfrak{m}}\right)\right)$ is an Artinian $R_{\mathfrak{m}}$-module and hence has a natural structure as an $\widehat{R_{\mathfrak{m}}}$-module.

The following theorem is a slight generalization of [22, Theorem 3.3].

Theorem 4.9. The following are equivalent:

(i) $C$ is pointwise dualizing.

(ii) If $M$ is a cotorsion $R$-module such that $C$-id ${ }_{R}(M)=n<\infty$, then $M$ admits a minimal flat resolution such that $\pi_{i}(\mathfrak{p}, M)=0$ for all $\mathfrak{p} \in \operatorname{Spec}(R)$ whenever ht $(\mathfrak{p}) \notin\{i, \ldots, i+n\}$.

Proof. (i) $\Longrightarrow$ (ii). We use induction on $n$. If $n=0$, then we are done by Theorem 4.3. Now assume inductively that $n>0$ and the case $n$ ie settled. Fix a prime ideal $\mathfrak{p}$ of $R$. Assume that $M$ is a cotorsion $R$-module with $C$-id $R(M)=n+1$. Hence $M \in \mathcal{A}_{C}(R)$, and so the $\mathcal{I}_{C}$-preenvelope of $M$ is injective by [18, Corollary 2.4(b)]. Thus there exists an exact sequence

$$
0 \rightarrow M \rightarrow \operatorname{Hom}_{R}(C, I) \rightarrow L \rightarrow 0,(*)
$$

in which $I$ is injective, and $L=\operatorname{Coker}\left(M \rightarrow \operatorname{Hom}_{R}(C, I)\right)$. Note that $L$ is cotorsion since both $M$ and $\operatorname{Hom}_{R}(C, I)$ are cotorsion. Also, since both $M$ and $\operatorname{Hom}_{R}(C, I)$ are in $\mathcal{A}_{C}(R)$, we have $L \in \mathcal{A}_{C}(R)$, and therefore $\operatorname{Tor}_{1}^{R}(C, L)=0$. On the other hand 
$C \otimes_{R} \operatorname{Hom}_{R}(C, I) \cong I$, by [7, Theorem 3.2.11]. Hence application of $C \otimes_{R}-$ on (*) yields an exact sequence

$$
0 \rightarrow C \otimes_{R} M \rightarrow I \rightarrow C \otimes_{R} L \rightarrow 0
$$

By Theorem 2.9(i), we have id ${ }_{R}\left(C \otimes_{R} M\right)=n+1$. Therefore id ${ }_{R}\left(C \otimes_{R} L\right)=n$, whence $C$-id ${ }_{R}(L)=n$. Now induction hypothesis applied to $\operatorname{Hom}_{R}(C, I)$ and $L$ yields that $\pi_{i}\left(\mathfrak{p}, \operatorname{Hom}_{R}(C, I)\right)=0$ for all $i \neq$ ht $(\mathfrak{p})$, and that $\pi_{i}(\mathfrak{p}, L)=0$ fo all ht $(\mathfrak{p}) \notin\{i, \ldots, i+n\}$. Note that $\operatorname{Ext}_{R}^{1}\left(R_{\mathfrak{p}}, M\right)=0$ since $M$ is cotorsion. Hence the exact sequence (*) yields an exact sequence

$$
0 \rightarrow \operatorname{Hom}_{R}\left(R_{\mathfrak{p}}, M\right) \rightarrow \operatorname{Hom}_{R}\left(R_{\mathfrak{p}}, \operatorname{Hom}_{R}(C, I)\right) \rightarrow \operatorname{Hom}_{R}\left(R_{\mathfrak{p}}, L\right) \rightarrow 0
$$

and the later exact sequence, by applying $k(\mathfrak{p}) \otimes_{R_{\mathfrak{p}}}-$, yields the long exact sequence

$$
\begin{gathered}
\cdots \rightarrow \operatorname{Tor}_{i+1}^{R_{\mathfrak{p}}}\left(k(\mathfrak{p}), \operatorname{Hom}_{R}\left(R_{\mathfrak{p}}, \operatorname{Hom}_{R}(C, E)\right)\right) \rightarrow \operatorname{Tor}_{i+1}^{R_{\mathfrak{p}}}\left(k(\mathfrak{p}), \operatorname{Hom}_{R}\left(R_{\mathfrak{p}}, L\right)\right) \rightarrow \\
\operatorname{Tor}_{i}^{R_{\mathfrak{p}}}\left(k(\mathfrak{p}), \operatorname{Hom}_{R}\left(R_{\mathfrak{p}}, M\right)\right) \rightarrow \operatorname{Tor}_{i}^{R_{\mathfrak{p}}}\left(k(\mathfrak{p}), \operatorname{Hom}_{R}\left(R_{\mathfrak{p}}, \operatorname{Hom}_{R}(C, E)\right)\right) \rightarrow \cdots
\end{gathered}
$$

From the above long exact sequence, it follows that $\operatorname{Tor}_{i}^{R_{\mathfrak{p}}}\left(k(\mathfrak{p}), \operatorname{Hom}_{R}\left(R_{\mathfrak{p}}, M\right)\right)=0$ for all ht $(\mathfrak{p}) \notin\{i, \ldots, i+n+1\}$, as wanted. This completes the inductive step.

(ii) $\Longrightarrow$ (i). Let $\mathfrak{m}$ be a maximal ideal of $R$. Now $\operatorname{Hom}_{R}(C, E(R / \mathfrak{m}))$ is $C$-injective and hence by assumption $\pi_{i}\left(\mathfrak{m}, \operatorname{Hom}_{R}(C, E(R / \mathfrak{m}))\right)=0$ for all $i \neq \mathrm{ht}(\mathfrak{m})$. Now by the same argument as in the proof of Theorem 4.3, we have $\operatorname{Ext}_{R_{\mathfrak{m}}}^{i}\left(k(\mathfrak{m}), C_{\mathfrak{m}}\right)=0$ for all $i \neq \mathrm{ht}(\mathfrak{m})$, whence $C_{\mathfrak{m}}$ is dualizing for $R_{\mathfrak{m}}$.

Corollary 4.10. The following statements hold true:

(i) If $C$ is pointwise dualizing, then $C$-id ${ }_{R}(F(M)) \leq C$-id ${ }_{R}(M)$ for any cotorsion $R$ module $M$.

(ii) If $C$-id ${ }_{R}(F(M)) \leq C$-id ${ }_{R}(M)$ for any $R$-module $M$, then $C$ is pointwise dualizing.

Proof. (i). Assume that $M$ is a cotorsion $R$-module. If $C$-id $R_{R}(M)=\infty$, then we are done. So assume that $C$-id ${ }_{R}(M)=n<\infty$. Then by Theorem 4.9, we have $F(M)=\prod T_{\mathfrak{p}}$ where $0 \leq \mathrm{ht}(\mathfrak{p}) \leq n$. Now the result follows by Lemma 4.1 .

(ii). Assume that $\mathfrak{m}$ is a maximal ideal of $R$. We have to show that $C_{\mathfrak{m}}$ is dualizing for $R_{\mathfrak{m}}$. Assume that $\mathrm{x}$ is a maximal $R$-sequence in $\mathfrak{m}$. Then $\mathrm{fd}_{R}(R / \mathrm{x} R)<\infty$, and $\operatorname{Ass}_{R}(C / \mathbf{x} C)=\{\mathfrak{m}\}$ since $\mathbf{x}$ is also a maximal $C$-sequence. Hence we have the equalities

$$
\begin{aligned}
C \text {-fd }(C / \mathbf{x} C) & =\mathrm{fd}_{R}\left(\operatorname{Hom}_{R}(C, C / \mathbf{x} C)\right) \\
& =\mathrm{fd}_{R}\left(\operatorname{Hom}_{R}\left(C, C \otimes_{R} R / \mathbf{x} R\right)\right) \\
& =\mathrm{fd}_{R}(R / \mathbf{x} R) \\
& <\infty
\end{aligned}
$$

in which the first equality is from Theorem 2.9(ii), and the third one holds because $R / \mathrm{x} R \in$ $\mathcal{A}_{C}(R)$. Assume that $E$ is an injective cogenerator. Set $(-)^{\vee}=\operatorname{Hom}_{R}(-, E)$. Then $C$ $\operatorname{id}_{R}\left((C / \mathrm{x} C)^{\vee}\right)<\infty$ by Lemma 2.10(ii). Now if $F$ is the flat cover of $(C / \mathrm{x} C)^{\vee}$, then by assumption, we have $C$-id ${ }_{R}(F)<\infty$. Therefore, we have $C$ - $\mathrm{fd}_{R}\left(F^{\vee}\right)<\infty$ by Lemma 2.10(i). Next, note that we have

$$
C / \mathrm{x} C \hookrightarrow(C / \mathrm{x} C)^{\vee \vee} \hookrightarrow F^{\vee}
$$

Hence, the injective envelope of $C / \mathbf{x} C$ is a direct summand of $F^{\vee}$. Thus, in fact, $E(R / \mathfrak{m})$ 
is a direct summand of $F^{\vee}$, since $R / \mathfrak{m} \hookrightarrow C / \mathbf{x} C$. It follows that $C$ - $\operatorname{fd}_{R}(E(R / \mathfrak{m}))<\infty$, and hence we are done by Lemma 4.1 , since $\mathfrak{m}$ was arbitrary.

Acknowledgments. We thank the referee for very careful reading of the manuscript and also for his/her useful suggestions.

\section{REFERENCES}

1. L. Bican, R. El Bashir and E. Enochs, All modules have flat covers, Bull. London Math. Soc. 33 (2001), 385-390.

2. W. Bruns and J. Herzog, Cohen-Macaulay rings, Cambridge University Press, Cambridge, 1993.

3. M.P. Brodmann and R.Y. Sharp, Local cohomology: an algebraic introduction with geometric applications, Cambridge University Press, Cambridge, 1998.

4. L.W. Christensen, Gorenstien Dimensions, Lecture Notes in Math., vol. 1747, Springer, Berlin, 2000.

5. L.W. Christensen, Semi-dualizing complexes and their Auslander categories, Trans. Amer. Math. Soc. 5 (2001), 1839-1883.

6. E. Enochs, Flat covers and flat cotorsion modules, Proc. Amer. Math. Soc. 92 (1984), 179-184.

7. E. Enochs and O. Jenda, Relative Homological Algebra, de Gruyter Expositions in Mathematics 30, 2000.

8. E. Enochs and J. Xu, On invariants dual to the Bass numbers, Proc. Amer. Math. Soc. 125 (1997), 951-960.

9. H.-B. Foxby, Gorenstein modules and related modules, Math. Scand. 31 (1973), 267-284 .

10. E. S. Golod, G-dimension and generalized perfect ideals, Trudy Mat. Inst. Steklov. Algebraic geometry and its applications 165 (1984), 62-66.

11. R. Hartshorne, Local cohomology, A seminar given by A. Grothendieck, Harvard University, Fall, vol. 1961, Springer-Verlag, Berlin, 1967.

12. H. Holm, P. Jørgensen, Semi-dualizing modules and related Gorenstein homological dimensions, J. Pure Appl. Algebra, 205(2006) 423-445.

13. H. Holm, D. White, Foxby equivalence over associative rings, J. Math. Kyoto Univ. 47 no.4, (2007), 781-808.

14. B. Kubik, M. J. Leamer, and S. Sather-Wagstaff, Homology of artinian and Matlis reflexive modules I, J. Pure Appl. Algebra, 215 (2011), no. 10, 2486-2503.

15. B. Kubik, M. J. Leamer, and S. Sather-Wagstaff, Homology of artinian and mini-max modules, J. Algebra. 403 (2014), no.1, 229-272.

16. M. Rahmani and A.-J. Taherizadeh, Tensor product of $C$-injective modules, preprint, 2015, available from http://arxiv.org/abs/1503.05492

17. R. Takahashi, Some characterizations of Gorenstein local rings in terms of G-dimension, Acta Math. Hungar. 104 no. 4, (2004), 315-322.

18. R. Takahashi and D.White, Homological aspects of semidualizing modules, Math. Scand. 106 (2010) $5-10$.

19. M. Salimi, E. Tavasoli, S. Sather-Wagstaff and S. Yassemi, Relative tor functor with respect to a semidualizing module, Algebras and Representation Theory, 17, no.1, (2014), 103-120.

20. W. V. Vasconcelos, Divisor theory in module categories, North-Holland Math. Stud. 14, North-Holland Publishing Co., Amsterdam (1974).

21. J. Xu, Flat covers of modules, Lecture Notes in Mathematics, Vol. 1634, Springer, New York, (1996).

22. J. Xu, Minimal injective and flat resolutions of modules over Gorenstein rings, J. Algebra 175 (1995), 451-477.

23. S. Yassemi, G-dimension, Math. Scand. 77 (1995), no. 2, 161-174. 
Faculty of Mathematical Sciences and Computer, Kharazmi University, Tehran, Iran.

E-mail address: m.rahmani.math@gmail.com

E-mail address: taheri@khu.ac.ir 